\title{
EXPERIMENTATION TO PREDICT THE THERMAL PERFORMANCE OF CONVENTIONAL HEAT PIPE WITH WATER AND HYDROCARBON AS WORKING FLUID
}

\author{
Roshan D. Bhagat ${ }^{1}$, Sashikant R. Thakare ${ }^{2}$, Sanjaykumar C. Makwana ${ }^{3}$ \\ ${ }^{l}$ Assistant Professor, Department of Mechanical Engineering, College of Engineering And Technology, Akola, \\ Sant Gadge Baba Amravati University, Maharashtra, India. \\ roshan.bhagat25@gmail.com \\ ${ }^{2}$ Assistant Professor, Department of Mechanical Engineering, College of Engineering And Technology, Akola, \\ Sant Gadge Baba Amravati University, Maharashtra, India. \\ shashi_thakare@rediffmail.com \\ ${ }^{3}$ Associate Professor, Department of Mechanical Engineering, College of Engineering And Technology, Akola, \\ Sant Gadge Baba Amravati University, Maharashtra, India. \\ sanjaykumarmakwana@gmail.com
}

\begin{abstract}
This work attempts to analyses the performance of conventional heat pipe with water and hydrocarbon as working fluid. The hydrocarbon working fluid involve acetone and methanol. The experimental investigation involves the determination of thermal resistance of conventional heat pipe at various heat input and to determine the best working fluid out of the water, acetone and methanol. Conventional heat pipe is filled with water, acetone and methanol with the filling ratio of $60 \%$ with this filling ratio the thermal performance of the device is investigated.
\end{abstract}

Keywords: Working Fluid, Heat Transfer, Thermal Resistance, Thermal Performance.

$* * *$

\section{INTROUDUCTION}

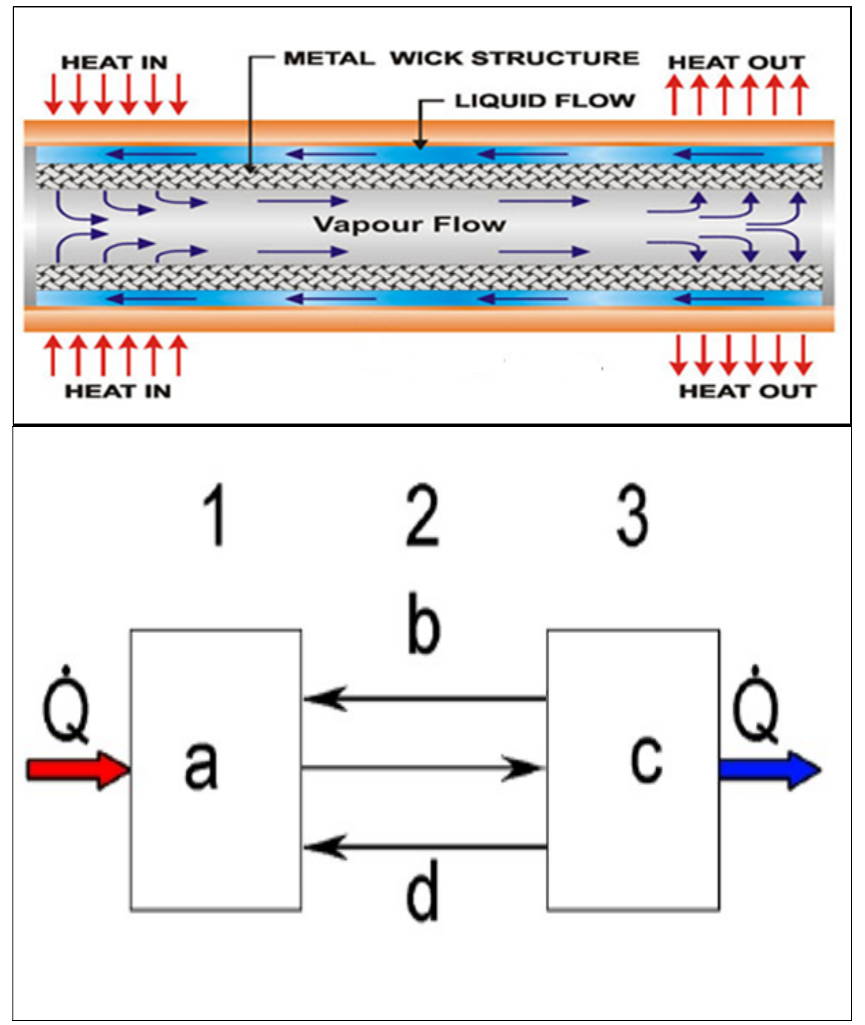

Fig. 1: Conventional heat pipe and its transport process

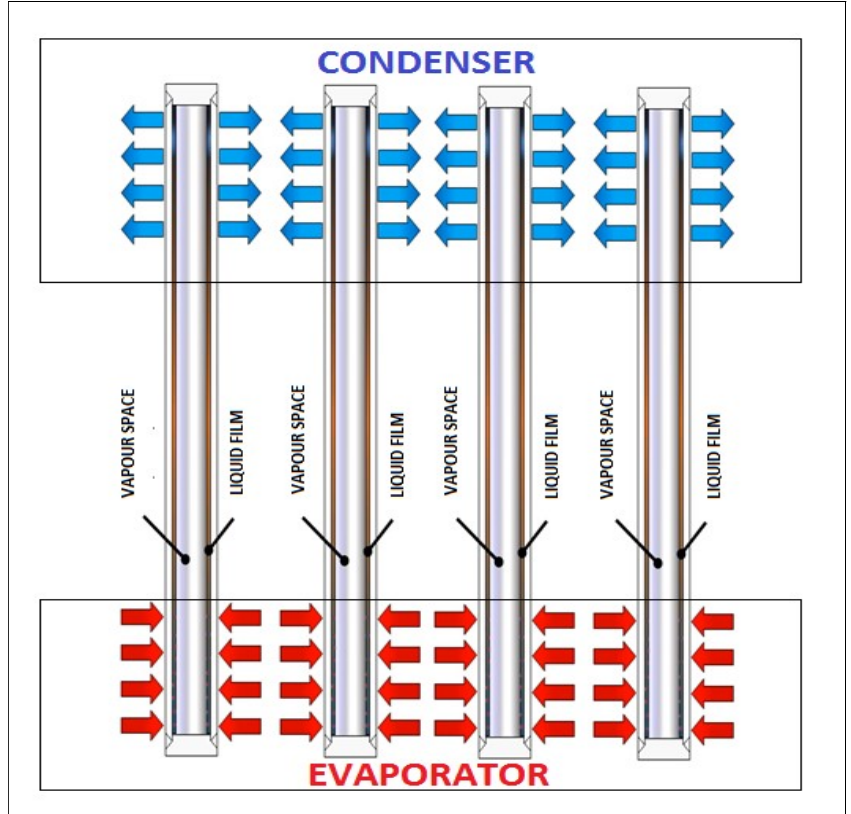

Fig. 2: Conventional heat pipe with evaporator and condenser section

The purpose of this initiative was to combine fluid dynamics and heat transfer to create a device capable of transferring heat within the small distance. The first phase focused on fabricating the apparatus and performing the initial testing to 
gain preliminary insight into its functionality. In addition to successfully transferring heat from heat, this task provided an opportunity for experimental learning and device creation and targeted design through engineering principles.

\subsection{Setup Description}

\subsubsection{Working Fluid}

Working fluid significantly influence on the thermal performance of heat pipe. Hydrocarbon working fluid involves Acetone, Methanol, Ethanol, Methane and Pentane. Experimental setup consists of conventional heat pipe with hydrocarbon as working fluid. The boiling point of Methanol is $64^{\circ} \mathrm{C}$ and Acetone $57^{\circ} \mathrm{C}, 60 \%$ filling ratio has been used for Acetone and Methanol.

\subsubsection{Copper Tube}

Copper tube is used for preparing the experimental setup. The diameter of copper tube is $8 \mathrm{~mm}$.

\subsubsection{Digital Laser Thermometer}

For measuring the evaporator and condenser temperature digital LASER thermometer is used. The digital LASER thermometer provides to flexibility to measure the temperature over the entire length of the copper tube.

\subsubsection{Evaporator Tank}

The evaporator tank design to the dimension of 8 inch $\times 6$ inch $\times 4$ inch, so as to occupy the heating element and to have sufficient amount of water inside the evaporator tank for heating the copper tube, with the water bath heating is done.

\subsubsection{Condenser Tank}

The dimension of condenser tank is taken as 8 inch $\times 3$ inch $\times 3$ inch. The condenser tank should hold sufficient water so as to have heat rejection by the working fluid through the copper tube and to have condensation of working fluid.

\subsubsection{Coil Heater}

The coil heater with capacity of 500 Watt is used to heat the water inside the evaporator tank.

\subsubsection{Temperature Indicator For Water Bath In}

\section{Evaporator}

For measuring the water bath temperature inside the evaporator tank, temperature indicator is used to monitor the temperature of water bath so as to prevent the excessive heating of water which may cause the dry out of working fluid in the copper tube.

\subsubsection{Variable Ac Power Supply}

For changing the heat inputs to the coil heater variable AC power supply is used. 0-240 VAC can be adjusted with the help of dimmerstat. Digital voltmeter and ammeter are connected to show the voltage and current reading.

\subsubsection{Control Panel}

Digital voltmeter, ammeter and water bath temperature indicator are mounted on the control panel.

\subsubsection{Tank Material}

Evaporator and condenser tank are prepared with acrylic. Acrylic is non conducting material and heat loss to the surrounding is kept minimum. Magnabond instant adhesive is used for preparing leak proof tank.

\subsection{Experimentation and Testing Of Conventional}

\section{Heat Pipe with Hydrocarbon As Working Fluid.}

The experimentation performed on conventional heat pipe by heating the water bath with the help of coil heater. The heat inputs to the coil heater can be adjusted by using 0 to 240 VAC power supply. Heat transfer by convection takes place from water to the copper tube and heat is conducted through the copper tube, this heat is now transferred to the working fluid present inside the copper tube.

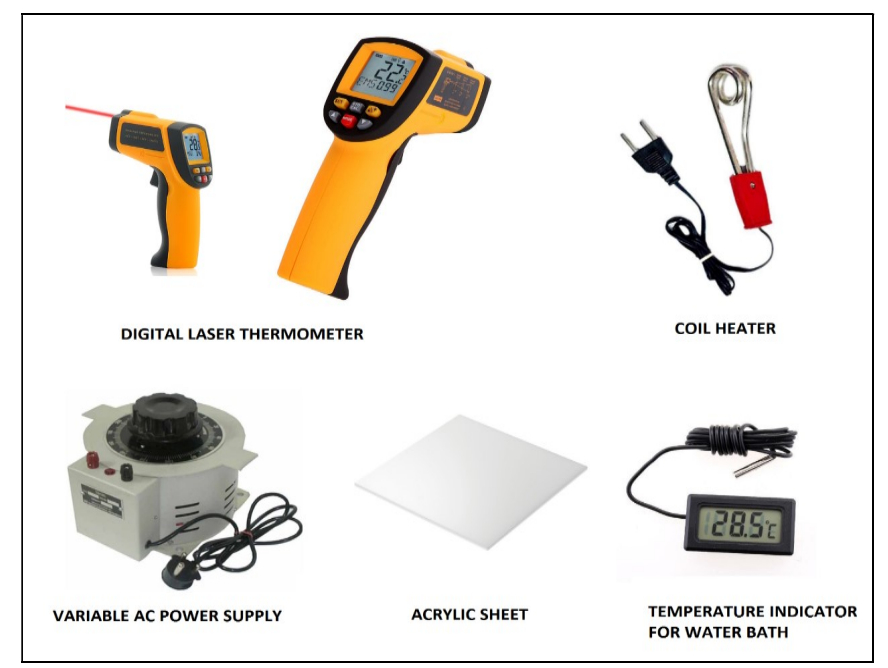

Fig 3: Equipment's used in experimental setup of conventional heat pipe with Hydrocarbon as working fluid

\subsection{Calculation}

The thermal resistance of CLPHP can be calculated by using the following equations,

$R_{\text {thermal }}=\frac{T_{e}-T_{c}}{Q}$ $\ldots \theta q^{n}(1)$

$R_{\text {thermal }}$ - Thermal resistance

$T_{f}$ - Average evaporator temperature

$T_{c}-$ Average condenser temperature

$Q-$ Heat input

$Q=$ Voltage $\times$ Current

Average evaporator temperature is calculated by using the equation $T_{e}=\frac{I_{1}^{*}+I_{2}+T_{a}^{*}+I_{4}^{*}+T_{5}^{*}+T_{6}^{*}}{6}$ $\ldots \theta q^{n}(3)$

Average condenser temperature is calculated by using the equation $T_{0}=\frac{T_{7}+T_{g}+T_{9}^{*}+T_{10}^{*}+T_{11}^{*}+T_{12}^{*}}{6} \quad \quad \ldots e q^{n}$ (4) 
Table 1: Evaporator and condenser temperature of conventional heat pipe with Acetone as working fluid.

\begin{tabular}{|c|c|c|c|c|c|c|c|c|c|c|c|c|c|c|}
\hline \multirow[t]{2}{*}{ S.N. } & \multirow[t]{2}{*}{$\mathbf{V}$} & \multirow[t]{2}{*}{ I } & \multirow[t]{2}{*}{$\mathbf{Q}$} & \multicolumn{4}{|c|}{$\begin{array}{l}\text { EVAPORATOR } \\
\text { TEMPERATURE } \\
\text { OF ACETONE } \bar{i} \eta^{0} G\end{array}$} & \multicolumn{4}{|c|}{$\begin{array}{l}\text { CONDENSER } \\
\text { TEMPERATURE OF } \\
\text { ACETONE } \bar{i} n^{*} \mathrm{C}\end{array}$} & \multirow[t]{2}{*}{ 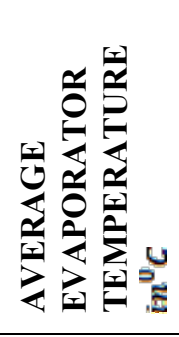 } & \multirow[t]{2}{*}{ 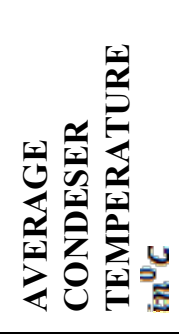 } & \multirow[t]{2}{*}{ 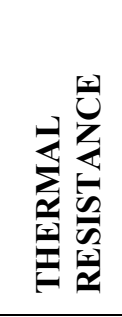 } \\
\hline & & & & T1 & T2 & T3 & T4 & T5 & T6 & $\mathrm{T} 7$ & T8 & & & \\
\hline 1 & 70 & 0.87 & 60.9 & 28.7 & 32 & 31 & 31.3 & 29.9 & 30.2 & 30.3 & 30.2 & 30.75 & 30.15 & 0.00985 \\
\hline 2 & 75 & 0.93 & 69.75 & 29.8 & 32.8 & 32.5 & 32.5 & 30.6 & 30.4 & 30.5 & 30.5 & 31.9 & 30.5 & 0.02007 \\
\hline 3 & 80 & 1 & 80 & 31.5 & 33.6 & 32.5 & 31.8 & 30.3 & 30.4 & 30.6 & 30.6 & 32.35 & 30.475 & 0.02344 \\
\hline 4 & 85 & 1.06 & 90.1 & 32.5 & 33.7 & 33.8 & 34.1 & 30.4 & 30.5 & 30.7 & 30.7 & 33.525 & 30.575 & 0.03274 \\
\hline 5 & 90 & 1.11 & 99.9 & 31.1 & 34.3 & 34.9 & 34.9 & 30.6 & 30.8 & 30.7 & 30.8 & 33.8 & 30.725 & 0.03078 \\
\hline 6 & 95 & 1.18 & 112.1 & 32.4 & 34.8 & 36.3 & 36.3 & 30.9 & 30.8 & 31 & 30.9 & 34.95 & 30.9 & 0.03613 \\
\hline 7 & 100 & 1.23 & 123 & 33.5 & 33.7 & 35.7 & 37.8 & 30.9 & 30.8 & 30.8 & 30.8 & 35.175 & 30.825 & 0.03537 \\
\hline 8 & 105 & 1.3 & 136.5 & 33.6 & 37 & 37 & 36.6 & 30.7 & 31 & 31.1 & 31 & 36.05 & 30.95 & 0.03736 \\
\hline 9 & 110 & 1.36 & 149.6 & 33.1 & 37.7 & 39 & 37.6 & 30.9 & 31 & 31 & 31.1 & 36.85 & 31 & 0.0391 \\
\hline 10 & 115 & 1.42 & 163.3 & 34.4 & 37.5 & 37.8 & 39 & 31 & 31 & 31.1 & 31.2 & 37.175 & 31.075 & 0.03735 \\
\hline 11 & 120 & 1.48 & 177.6 & 35.3 & 39.3 & 39 & 38.8 & 31.1 & 31.1 & 31.1 & 31.1 & 38.1 & 31.1 & 0.03941 \\
\hline 12 & 125 & 1.54 & 192.5 & 37.5 & 41.6 & 40.5 & 40.1 & 31.1 & 31.2 & 31.3 & 31.3 & 39.925 & 31.225 & 0.04519 \\
\hline 13 & 130 & 1.61 & 209.3 & 38.2 & 41.1 & 41 & 40.8 & 31.4 & 31.4 & 32.1 & 31.7 & 40.275 & 31.65 & 0.04121 \\
\hline 14 & 135 & 1.67 & 225.45 & 42.6 & 44.3 & 44.9 & 44.7 & 31.4 & 31.4 & 31.5 & 31.4 & 44.125 & 31.425 & 0.05633 \\
\hline 15 & 140 & 1.73 & 242.2 & 40.3 & 43.2 & 44.7 & 44.5 & 31.3 & 31.4 & 31.5 & 31.5 & 43.175 & 31.425 & 0.04851 \\
\hline 16 & 145 & 1.8 & 261 & 42.8 & 44.9 & 46 & 45.5 & 31.5 & 31.4 & 31.4 & 31.5 & 44.8 & 31.45 & 0.05115 \\
\hline 17 & 150 & 1.85 & 277.5 & 42.3 & 45.2 & 45.2 & 45.1 & 31.8 & 31.6 & 31.8 & 31.7 & 44.45 & 31.725 & 0.04586 \\
\hline 18 & 155 & 1.91 & 296.05 & 41.9 & 45.2 & 46 & 44.5 & 31.8 & 31.8 & 31.9 & 31.7 & 44.4 & 31.8 & 0.04256 \\
\hline 19 & 160 & 1.98 & 316.8 & 41.5 & 47.5 & 47 & 47.4 & 32.1 & 31.9 & 32 & 32 & 45.85 & 32 & 0.04372 \\
\hline 20 & 165 & 2.05 & 338.25 & 47.1 & 50.7 & 50.5 & 50.7 & 32.1 & 32.1 & 32.3 & 32.3 & 49.75 & 32.2 & 0.05188 \\
\hline 21 & 170 & 2.1 & 357 & 46 & 52.3 & 51.2 & 50.7 & 32.6 & 32.6 & 32.8 & 32.8 & 50.05 & 32.7 & 0.0486 \\
\hline 22 & 175 & 2.16 & 378 & 50.6 & 52.6 & 51 & 51 & 32 & 32.9 & 33.1 & 33.1 & 51.3 & 32.775 & 0.04901 \\
\hline 23 & 180 & 2.2 & 396 & 46.3 & 52.7 & 52.7 & 52.1 & 33.2 & 33.1 & 32.9 & 33 & 50.95 & 33.05 & 0.0452 \\
\hline 24 & 185 & 2.28 & 421.8 & 53.2 & 57.2 & 56.4 & 55.3 & 33.5 & 33.3 & 33.9 & 33.8 & 55.525 & 33.625 & 0.05192 \\
\hline 25 & 190 & 2.34 & 444.6 & 51.5 & 57.7 & 57.3 & 57.6 & 33.4 & 33.4 & 33.4 & 33.5 & 56.025 & 33.425 & 0.05083 \\
\hline 26 & 195 & 2.41 & 469.95 & 52.3 & 59.5 & 57.5 & 57.2 & 33.5 & 33.5 & 33.7 & 33.8 & 56.625 & 33.625 & 0.04894 \\
\hline 27 & 200 & 2.45 & 490 & 49.8 & 58.8 & 57.7 & 57.7 & 34 & 33.5 & 34.9 & 33.9 & 56 & 34.075 & 0.04474 \\
\hline 28 & 205 & 2.5 & 512.5 & 51.6 & 60 & 58.7 & 59 & 33.7 & 33.8 & 33.9 & 33.9 & 57.325 & 33.825 & 0.04585 \\
\hline 29 & 210 & 2.55 & 535.5 & 56.8 & 63.2 & 62.6 & 63.3 & 35.4 & 34.3 & 34.9 & 35.1 & 61.475 & 34.925 & 0.04958 \\
\hline
\end{tabular}


Table 2: Evaporator and condenser temperature of conventional heat pipe with Methanol as working fluid.

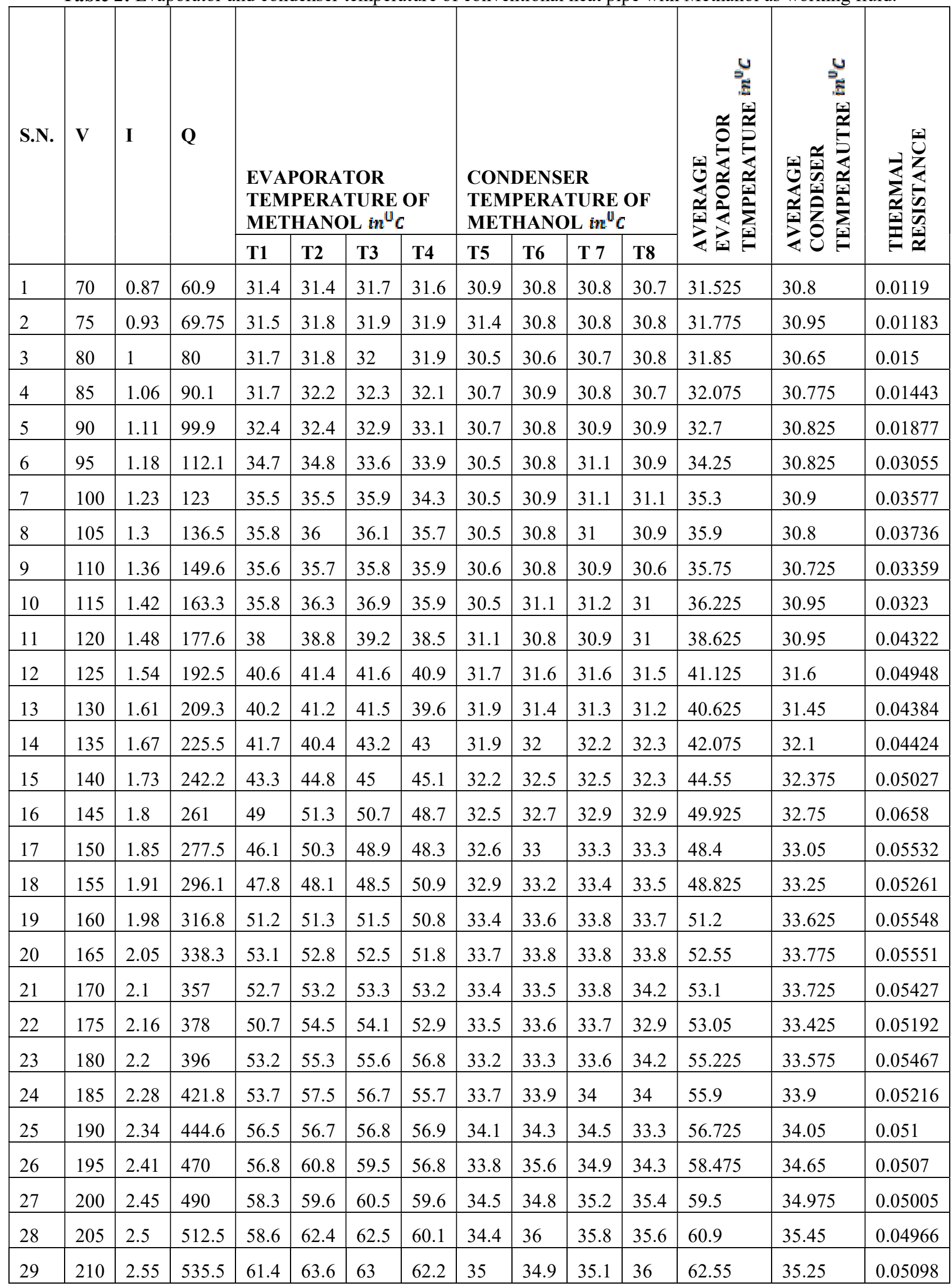


Table 3: Evaporator and condenser temperature of conventional heat pipe with Water as working fluid.

\begin{tabular}{|c|c|c|c|c|c|c|c|c|c|c|c|c|c|c|}
\hline \multirow[t]{2}{*}{ S.N. } & \multirow[t]{2}{*}{$\mathbf{V}$} & \multirow[t]{2}{*}{ I } & \multirow[t]{2}{*}{$\mathbf{Q}$} & \multicolumn{4}{|c|}{$\begin{array}{l}\text { EVAPORATOR } \\
\text { TEMPERATURE OF } \\
\text { WATER } i n^{0} C\end{array}$} & \multicolumn{4}{|c|}{$\begin{array}{l}\text { CONDENSER } \\
\text { TEMPERATURE OF } \\
\text { WATER } \text { in }^{*} C\end{array}$} & \multirow[t]{2}{*}{ 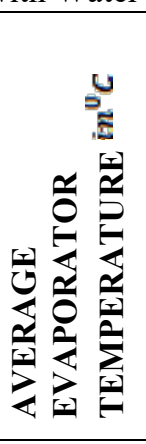 } & \multirow[t]{2}{*}{ 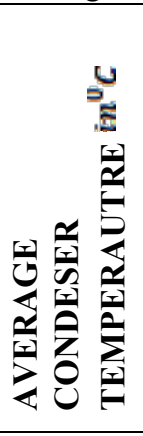 } & \multirow[t]{2}{*}{ 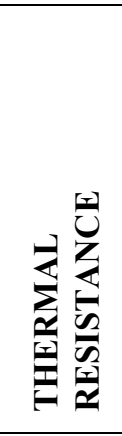 } \\
\hline & & & & T1 & $\mathbf{T 2}$ & T3 & T4 & T5 & T6 & $\mathbf{T} 7$ & T8 & & & \\
\hline 1 & 70 & 0.87 & 60.9 & 30.7 & 30.7 & 30.9 & 31 & 30.7 & 30.6 & 30.6 & 30.6 & 30.825 & 30.625 & 0.00328 \\
\hline 2 & 75 & 0.93 & 69.75 & 31.2 & 31.2 & 31.3 & 30.9 & 30.6 & 30.6 & 30.6 & 30.5 & 31.15 & 30.575 & 0.00824 \\
\hline 3 & 80 & 1 & 80 & 31.6 & 31.9 & 32 & 32.1 & 30.5 & 30.5 & 30.4 & 30.3 & 31.9 & 30.425 & 0.01844 \\
\hline 4 & 85 & 1.06 & 90.1 & 31.8 & 32.2 & 32.6 & 32.7 & 30.3 & 30.4 & 30.4 & 30.4 & 32.325 & 30.375 & 0.02164 \\
\hline 5 & 90 & 1.11 & 99.9 & 32.6 & 32.6 & 32.7 & 32.1 & 30.3 & 30.5 & 30.7 & 30.6 & 32.5 & 30.525 & 0.01977 \\
\hline 6 & 95 & 1.18 & 112.1 & 32.7 & 33.1 & 33.4 & 33.4 & 30.3 & 30.5 & 30.4 & 30.3 & 33.15 & 30.375 & 0.02475 \\
\hline 7 & 100 & 1.23 & 123 & 32.8 & 33.5 & 33.7 & 33.7 & 30.2 & 30.3 & 30.4 & 30.2 & 33.425 & 30.275 & 0.02561 \\
\hline 8 & 105 & 1.3 & 136.5 & 36.9 & 37.2 & 36.2 & 37.1 & 31.1 & 31.2 & 31.2 & 31.2 & 36.85 & 31.175 & 0.04158 \\
\hline 9 & 110 & 1.36 & 149.6 & 37.6 & 37.8 & 37.9 & 37.6 & 31.2 & 31 & 31.2 & 31 & 37.725 & 31.1 & 0.04428 \\
\hline 10 & 115 & 1.42 & 163.3 & 38.1 & 38.3 & 38.3 & 38.1 & 30.7 & 30.7 & 31.9 & 31.1 & 38.2 & 31.1 & 0.04348 \\
\hline 11 & 120 & 1.48 & 177.6 & 38.6 & 38.7 & 38.7 & 38.4 & 30.8 & 31 & 31.3 & 31.2 & 38.6 & 31.075 & 0.04237 \\
\hline 12 & 125 & 1.54 & 192.5 & 39.6 & 39.6 & 39.7 & 39.4 & 31 & 31.1 & 31.3 & 31.3 & 39.575 & 31.175 & 0.04364 \\
\hline 13 & 130 & 1.61 & 209.3 & 39.7 & 39.8 & 39.8 & 39.6 & 33.7 & 31.3 & 31.3 & 31.3 & 39.725 & 31.9 & 0.03739 \\
\hline 14 & 135 & 1.67 & 225.5 & 41.1 & 41.2 & 41.4 & 41.1 & 30.8 & 31.1 & 31.4 & 31.3 & 41.2 & 31.15 & 0.04458 \\
\hline 15 & 140 & 1.73 & 242.2 & 41.7 & 41.8 & 41.8 & 40.9 & 30.7 & 31.1 & 31.3 & 31.1 & 41.55 & 31.05 & 0.04335 \\
\hline 16 & 145 & 1.8 & 261 & 43.2 & 43.4 & 43.3 & 42.9 & 30.8 & 31.1 & 31.3 & 31 & 43.2 & 31.05 & 0.04655 \\
\hline 17 & 150 & 1.85 & 277.5 & 44.7 & 44.8 & 44.7 & 44.3 & 31.2 & 31.4 & 31.5 & 31.3 & 44.625 & 31.35 & 0.04784 \\
\hline 18 & 155 & 1.91 & 296.1 & 45.7 & 45.7 & 45.4 & 45.1 & 31.1 & 31.3 & 31.4 & 31.5 & 45.475 & 31.325 & 0.0478 \\
\hline 19 & 160 & 1.98 & 316.8 & 45.8 & 45.8 & 46.6 & 45.3 & 31.1 & 31.4 & 31 & 31 & 45.875 & 31.125 & 0.04656 \\
\hline 20 & 165 & 2.05 & 338.3 & 47 & 46.9 & 46.8 & 46.8 & 31.7 & 31.6 & 31.5 & 31.5 & 46.875 & 31.575 & 0.04523 \\
\hline 21 & 170 & 2.1 & 357 & 48.1 & 48.1 & 48.1 & 46.8 & 31.7 & 31.6 & 31.5 & 31.5 & 47.775 & 31.575 & 0.04538 \\
\hline 22 & 175 & 2.16 & 378 & 48.6 & 48.7 & 48.4 & 48.1 & 31.3 & 31.9 & 32 & 31.7 & 48.45 & 31.725 & 0.04425 \\
\hline 23 & 180 & 2.2 & 396 & 50.3 & 50.2 & 50.1 & 49.8 & 31.3 & 31.5 & 31.9 & 32 & 50.1 & 31.675 & 0.04653 \\
\hline 24 & 185 & 2.28 & 421.8 & 50.3 & 50.3 & 50.1 & 49.7 & 31.6 & 31.9 & 31.5 & 31.5 & 50.1 & 31.625 & 0.0438 \\
\hline 25 & 190 & 2.34 & 444.6 & 52.3 & 52.5 & 52.2 & 52 & 31.9 & 31.9 & 31.9 & 31.9 & 52.25 & 31.9 & 0.04577 \\
\hline 26 & 195 & 2.41 & 470 & 54.4 & 54.3 & 54.1 & 53.8 & 31.9 & 33.3 & 32.9 & 32.4 & 54.15 & 32.625 & 0.0458 \\
\hline 27 & 200 & 2.45 & 490 & 55.7 & 55.5 & 55.1 & 54.4 & 31.9 & 33.8 & 33.2 & 32.4 & 55.175 & 32.825 & 0.04561 \\
\hline 28 & 205 & 2.5 & 512.5 & 58.2 & 58.2 & 57.8 & 57.5 & 32.4 & 32.1 & 32.9 & 33.1 & 57.925 & 32.625 & 0.04937 \\
\hline 29 & 210 & 2.55 & 535.5 & 59.4 & 59.1 & 59.1 & 58.5 & 32.7 & 34.1 & 32.3 & 33 & 59.025 & 33.025 & 0.04855 \\
\hline
\end{tabular}




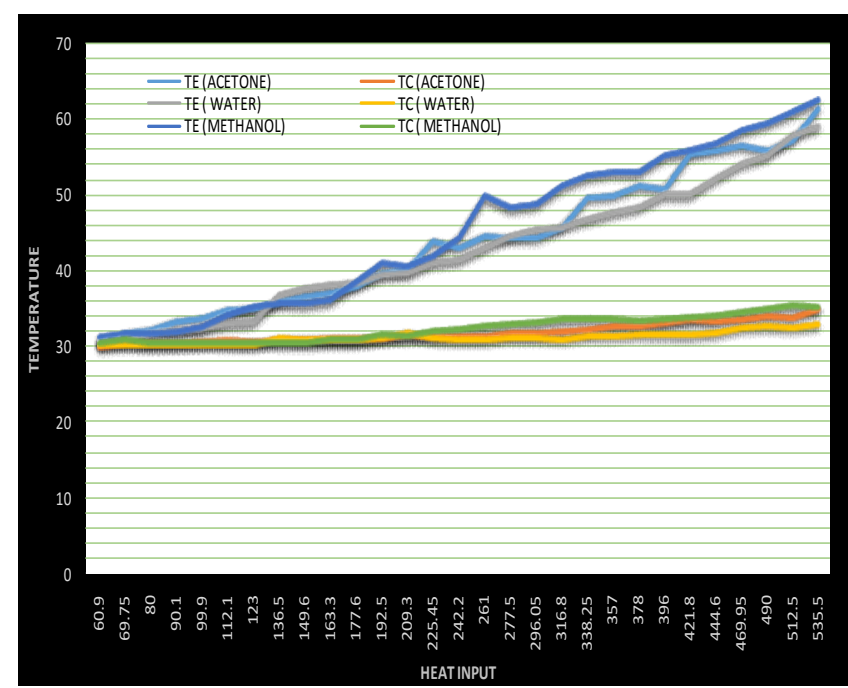

Fig.4: Evaporator and condenser tempeature of conventional heat pipe with Water and hydrocarbon as working fluid.

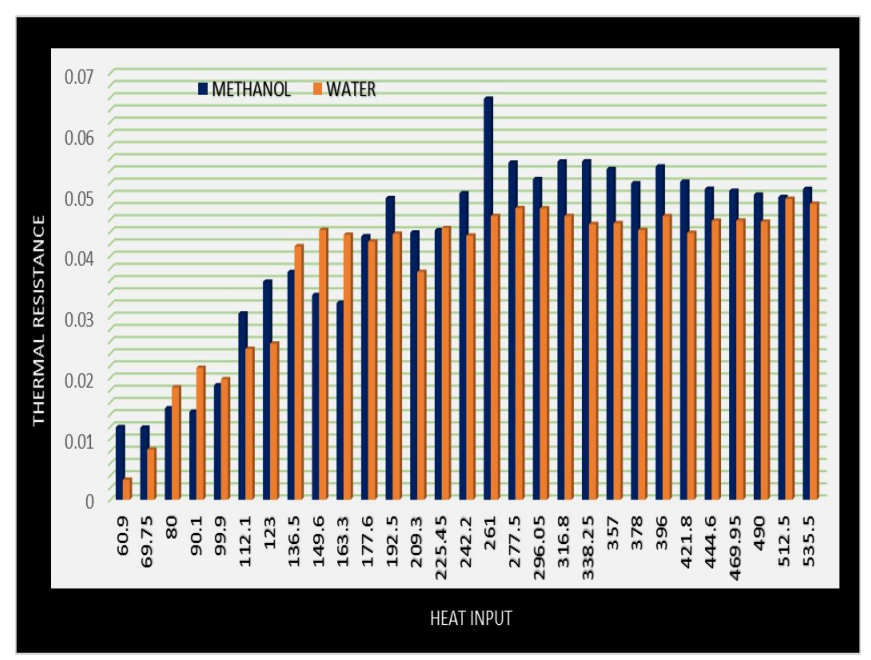

Fig. 5: Thermal resistance of conventional heat pipe with methanol and water as working fluid

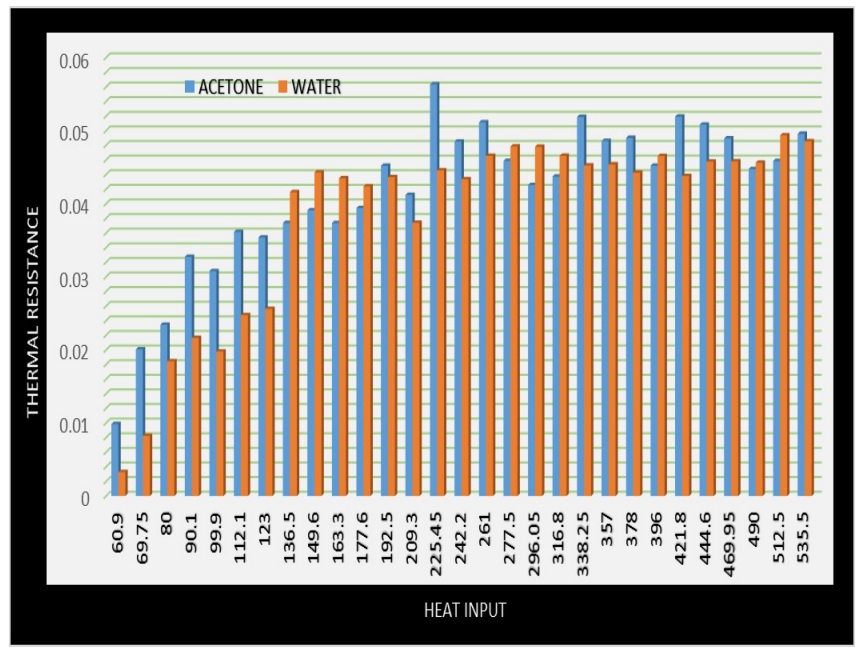

Fig. 6: Thermal resistance of conventional heat pipe with acetone and water as working fluid

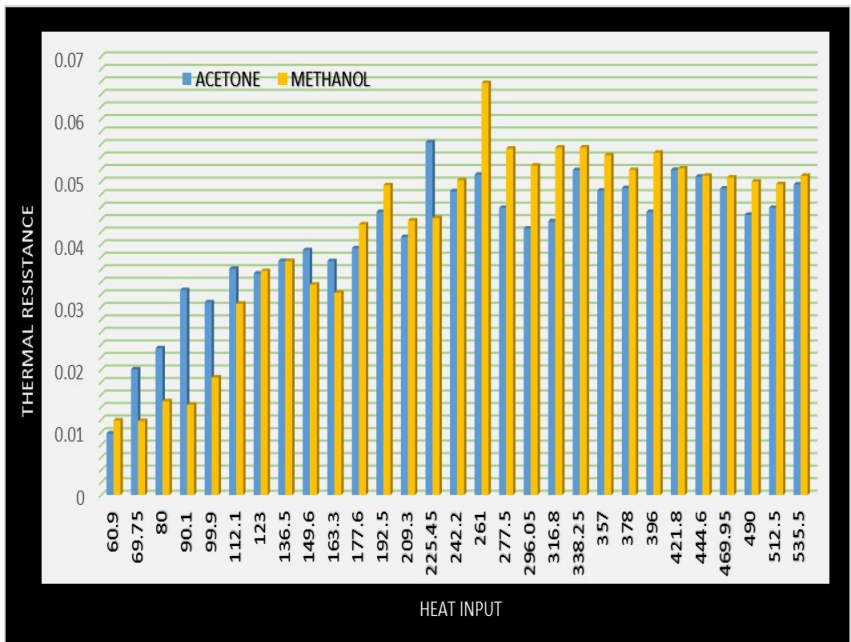

Fig. 7: Thermal resistance of conventional heat pipe with acetone and methanol as working fluid

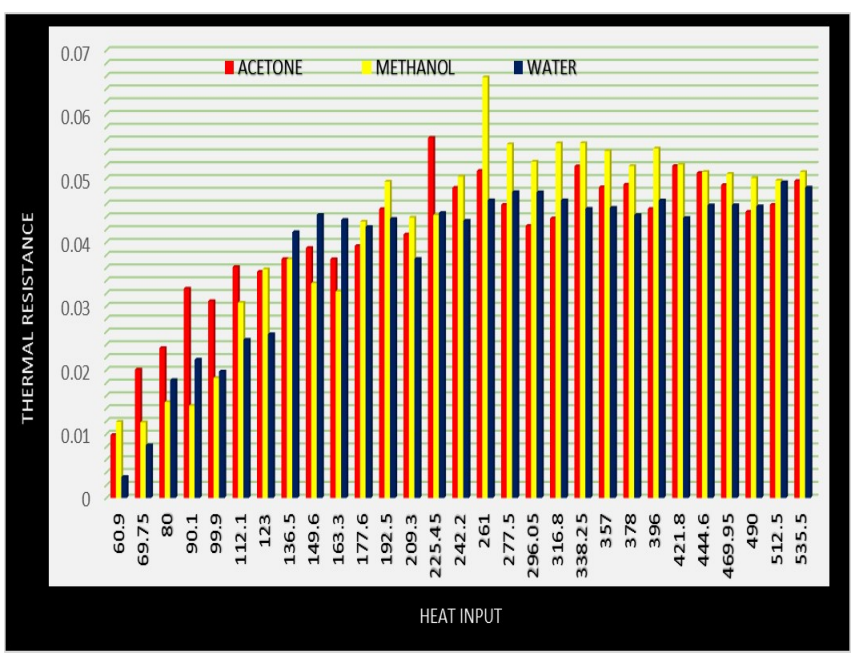

Fig. 8: Thermal resistance of conventional heat pipe with water and hydrocarbon as working fluid.

\section{RESULT AND DISCUSSION}

\subsection{RESULT}

$>\quad$ It was observed during the experimentation that as the water bath temperature increases there is increase in evaporator and condenser temperature of conventional heat pipe.

$>$ From fig. 5 it was observed that the thermal resistance of water is less than the thermal resistance of methanol hence water is suitable working fluid as compared to methanol.

$>$ From fig. 6 it was observed that the thermal resistance of water is less than the thermal resistance of acetone hence water is suitable working fluid as compared to acetone.

$>\quad$ Form fig.7 it was observed that at lower heat input the thermal resistance of acetone is higher than methanol whereas at higher heat input thermal resistance of acetone is lesser than that of methanol. 


\subsection{DISCUSSION}

The latent heat for Methanol is higher while the latent heat for Acetone is less, so the conventional heat pipe with Acetone as working fluid can be used for lower heat application because excessive heat can cause the dry out of working fluid. Whereas, Methanol can be used for higher heat applications. The advantage associated with Acetone due to lower latent heat is that it required less heat for converting liquid into vapour.

\subsection{SOURCES OF ERRORS}

$>\quad$ The temperature of tube is noted with the help of digital LASER thermometer, the accuracy of digital LASER thermometer depends on the distance to spot ratio. Hence, improper distance to spot ratio has possibility of creating errors while measurement.

Heat loss from water to the surrounding should be minimise to maximum possible extent to avoid deceptive evaporator and condenser readings.

Care should be taken to avoid excessive heat inputs which may cause drying out of working fluid in the tubes.

\section{CONCLUSION AND SCOPE FOR FUTURE}

\section{WORK}

\subsection{Conclusion}

The condenser temperature increases more rapidly when the water bath temperature reaches to the boiling point of working fluid used in conventional heat pipe. From the fig. 8 it was observed that the water is the best working fluid and it has lesser thermal resistance than the hydrocarbon working fluid like acetone and methanol. At all heat inputs the thermal performance of water proved to be better than any other working fluid. Hence at filling ratio of $60 \%$ out of the water, acetone and methanol the working fluid which provides best thermal performance is water.

\subsection{Scope For Future Work}

This works can be extended to future:

$>$ The same experimentation can be carried out at different filling ratio and by using other working fluids like Ethanol, Ammonia and R-123 etc.

$>\quad$ Thermal analysis of CLPHP using software packages like ANSYS.

\section{REFERENCES}

[1]. Incropera, F.P., DeWitt, D.P., Bergman, T.L., and Lavine, A.S., 2007, Fundamentals of Heat and Mass Transfer, $6^{\text {th }}$ ed., John Wiley \& Sons, Hoboken, NJ.

[2]. Khandekar S., Groll M., 2003, On the Definition of Pulsating Heat Pipe, Proceedings of $5^{\text {th }}$ Minsk International Seminar (Heat Pipes, Heat Pumps and Refrigerators), Minsk, Belarus.

[3]. Dadong Wang, Xiaoyu. Cui, 2010, Experimental Research on Pulsating Heat Pipe with Different
Mixtures Working Fluids, The $21^{s t}$ International Symposium on Transport Phenomena 2-5 November, Kaohsiung City, Taiwan.

[4]. P. K. Nag, Engineering Thermodynamics, McGraw Hill Education India Private Limited, Fifth Edition pp. 423-434.

[5]. Groll M., Khandekar S., 2002, Pulsating Heat Pipes: A Challenge and Still Unsolved Problem in Heat Pipe Science, Archives of Thermodynamics, ISSN 1231-0956, Vol. 23/4, pp. 17-28.

[6]. Nishio S., Nagata S., Baba S., Shirakashi R., 2002, Thermal Performance of SEMOS Heat Pipes, Proceedings of $12^{\text {th }}$ International Heat Transfer Conference, Grenoble, France, ISBN 2-84299-307-1, Vol. 4, pp.477-482.

[7]. S. Khandekar, M. Schneider, R. Kulenovic, M. Groll, Thermofluid Dynamic Study of Flat Plate CLPHPs, Microscience Thermophysics Engineering 6 (4) (2002) 303-318 (ISSN 1089-3954) http://dx.doi.org/10.1080/10893950290098340

[8]. M. B. Shafii, A. Faghri, Y. Zhang, Thermal Modeling of Unlooped and Looped Pulsating Heat Pipes, ASME Journal of Heat Transfer 123 (2001) 1159-1172. http://dx.doi.org/10.1115/1.1409266

[9]. Charoensawan P., Khandekar S. Groll M., 2004, Closed Loop and Open Loop Pulsating Heat Pipes, Proceedings of $13^{\text {th }}$ International Heat Pipe Conference, Shanghai, China, pp.21-25.

[10]. Khandekar S., Dollinger N., Groll M., Understanding Operational Regimes of Closed Loop Pulsating Heat Pipes: An Experimental Study, 2003, Applied Thermal Engineering, Vol. 23, pp.707-719. http://dx.doi.org/10.1016/S1359-4311(02)00237-5

[11]. Groll M., Khandekar S., 2004, An Insight into Thermo-Hydrodynamic Coupling in Closed Loop Pulsating Heat Pipes, International Journal of Thermal Sciences, Vol.43, pp.13-20. http://dx.doi.org/10.1016/S1290-0729(03)00100-5

[12]. M. Groll, S. Khandekar, Pulsating Heat Pipes: A Challenge and Still Unsolved Problem in Heat Pipe Science, Proceedings of $3^{\text {rd }}$ International Conference on Transport Phenomena in Multiphase Systems, Kielce, Poland, 2002, pp. 35-44 (ISBN 83- 8890603-8).

[13]. Arab M., Soltanieh M., Shafii M., 2012, Experimental Investigation of Extra-Long Pulsating Heat Pipe Application in Solar Water Heaters, Experimental Thermal and Fluid Science, http://dx.doi.org/10.1016/j.expthermflusci.2012.03.0 06

[14]. Khandekar S., Groll M., Charoensawan P., Terdtoon P., Pulsating Heat Pipes: Thermo-Fluidic Characteristics and Comparative Study with Single Phase Thermosyphon, Proceedings of $12^{\text {th }}$ International Heat Transfer Conference, ISBN-284299-307-1, Vol. 4, pp. 459-464, Grenoble, France, 2002.

[15]. Song Y. and Xu J, 2009, Chaotic Behaviour of Pulsating Heat Pipes, International Journal of Heat and Mass Transfer, Vol.52, pp.2932-294. 
http://dx.doi.org/10.1016/j.ijheatmasstransfer.2009.0 2.030

[16]. Narasimha K. R., Sridhara S.N., Rajagopal M.S., Seetharamu K.N., 2012, Influence of Heat Input, Working Fluid and Evacuation Level on the Performance of Pulsating Heat Pipe, Journal of Applied Fluid Mechanics, Vol. 5/2, pp. 33-42.

[17]. P. Charoensawan, S. Khandekar, M. Groll, P. Terdtoon, Closed Loop Pulsating Heat Pipes- Part A: Parametric Experimental Investigations, Applied Thermal Engineering, Vol. 23, no. 16, pp. 20092020, 2003. http://dx.doi.org/10.1016/s13594311(03)00159-5

[18]. S. Khandekar, P. Charoensawan, M. Groll, P. Terdtoon, Closed Loop Pulsating Heat Pipes- Part B: Visualization and Semi-Empirical Modeling, Applied Thermal Engineering, Vol. 23, no. 16, pp. 20212033, 2003. http://dx.doi.org/10.1016/s13594311(03)00168-6

[19]. H. Akachi, F. Polasek, P. Stulc, Pulsating Heat Pipes, Proceedings of $5^{\text {th }}$ International Heat Pipe Symposium, Melbourne, Australia, 1996, pp. 208217.

[20]. N. Soponpongpipat, P. Sakulchangsatjatai, N. Kammuanglue, P. Terdtoon, Investigation of the Start-up Condition of a Closed Loop Oscillating Heat Pipe, Heat Transfer Engineering, Vol. 30, no. 8, pp. 626-642, 2009.http://dx.doi.org/10.1080/01457630802656876

[21]. Y. Zhang, Amir Faghri, Heat Transfer in a Pulsating Heat Pipe with Open End, Heat Mass Transfer, Vol. 45, pp. 755-764, 2002. http://dx.doi.org/10.1016/s0017-9310(01)00203-4

[22]. M. B. Shafii, A. Faghri, Y. Zhang, Analysis of Heat Transfer in Unlooped and Looped Pulsating Heat Pipes, International Journal of Numerical Methods for Heat and Fluid Flow, Vol. 12, no. 5, pp. 585-609, 2002. http://dx.doi.org/10.1108/09615530210434304

[23]. Tong B.Y., Wong T.N., Ooi K.T., 2001, Closed Loop Pulsating Heat Pipe, Applied Thermal Engineering, ISSN 1359-4311, Vol. 21/18, pp. 1845-1862. http://dx.doi.org/10.1016/S1359-4311(01)00063-1

[24]. C. P. Kothandaraman, S. Subramanyam, Heat and Mass Transfer Data Book (2014), Eight Edition, New Age International Publishers pp 189-190.

[25]. Amir Faghri, Heat Pipes: Review, Opportunities and Challenges, Frontiers in Heat Pipes (FHP), (2014), Global Digital Central, ISSN: 2155-658X, http://dx.doi.org10.5098/fhp.5.1

[26]. Roshan D. Bhagat, Prof. K.M.Watt, Closed Loop Pulsating Heat Pipe with Hydrocarbon as Working Fluid: A Review, International Journal of Science and Research (IJSR) ISSN (Online): 2319-7064 Impact Factor (2012): 3.358, Volume 3 Issue 10, October 2014, Available @ http://www.ijsr.net.

[27]. Roshan D. Bhagat, Prof. K.M.Watt, Effect of Dimensionless Number on Thermal Performance of Closed Loop Pulsating Heat Pipe: A Review, International Journal of Science and Research (IJSR) ISSN (Online): 2319-7064 Index Copernicus Value
(2013): 6.14 | Impact Factor (2013): 4.438, Volume 4 Issue 2, February 2015, Available @ http://www.ijsr.net.

[28]. Roshan D. Bhagat, Prof. K.M.Watt, An Experimental Investigation of Methanol Closed Loop Pulsating Heat Pipe at Variable Water Bath Temperature, International Journal of Science and Research (IJSR) ISSN (Online): 2319-7064 Index Copernicus Value (2013): 6.14 | Impact Factor (2013): 4.438, Volume 4 Issue 2, February 2015, Available @ http://www.ijsr.net.

[29]. Roshan D. Bhagat, Prof. K.M.Watt, Effect of Water Bath Temperature on Evaporator and Condenser Temperature of Closed Loop Pulsating Heat Pipe With Acetone as Working Fluid, International Journal for Research in Engineering and Technology (IJRET) eISSN: 2319-1163| pISSN:2321-7308, Impact Factor (2013): 2.375, Volume 4 Issue 2, February 2015, Available@ http://www.ijret.org. http://dx.doi.org/10.15623/ijret.2015.0402050

[30]. Roshan D. Bhagat, Prof. K.M.Watt, Experimentation to Predict the Thermal Perfomance of Closed Loop Pulsating Heat Pipe with Acetone and Methanol as Working Fluid, International Journal for Research in Engineering and Technology (IJRET) eISSN: 23191163| pISSN:2321-7308, Impact Factor (2013): 2.375, Volume 4 Issue 4, April 2015, Available@http://www.ijret.org .http://dx.doi.org/10.15623/ijret.2015.0404005

[31]. Roshan D. Bhagat, Prof. K.M.Watt, Performance Investigation of Closed Loop Pulsating Heat Pipe with Acetone as Working Fluid, International Journal for Research in Engineering and Technology (IJRET) eISSN: 2319-1163| pISSN:2321-7308, Impact Factor (2013): 2.375, Volume 4 Issue 4, April 2015, Available @ @ http://www.ijret.org. http://dx.doi.org/10.15623/ijret.2015.0404001

[32]. Roshan D. Bhagat, Prof. K.M.Watt, Performance Investigation of Closed Loop Pulsating Heat Pipe with Methanol as Working Fluid, Proceedings of Mechanical Engineering Post Graduate Conference at PRMIT \& R Badnera-Amravati, MEPCON-2015. International Journal of Innovative and Emerging Research in Engineering, eISSN: 2394-3343| pISSN:2394-5494, Volume 2, Special Issue 1 MEPCON 2015, Available@ @ttp://www.ijiere.com.

[33]. Roshan D. Bhagat, Prof. K.M.Watt, Effect of Bond Number on Thermophysical Properties of Working Fluid used in Closed Loop Pulsating Heat Pipe: A Review, International Journal of Science and Research (IJSR) ISSN (Online): 2319-7064 Index Copernicus Value (2013): 6.14 | Impact Factor (2013): 4.438, Volume 4 Issue 9, September 2015, Available@http://www.ijsr.net. 